\title{
Erratum zu: Der Kontaktbogen: Gute Vorbereitung als Voraussetzung für erfolgreiches Nachfassen
}

\section{Erratum zu:}

\section{Kapitel 8 In: T. Dehghan, Der Messecoach,}

https://doi.org/10.1007/978-3-658-22765-4_8

Trotz sorgfältiger Erstellung unserer Bücher lassen sich Fehler nicht vermeiden, daher weisen wir auf Folgendes hin:

Die Originalversion des Kapitels wurde revidiert. Im Inhaltsverzeichnis und in Abschnitt 8.2 wurde die Abschnittsüberschrift korrigiert. 